\title{
Techniques for Improving Buried Mine Detection in Thermal IR Imagery
}

\author{
İbrahim Kürşat Şendur and Brian A. Baertlein \\ The Ohio State University, ElectroScience Laboratory \\ 1320 Kinnear Road, Columbus, OH 43212
}

\begin{abstract}
We describe sensor-based and signal-processing-based techniques for improving the detection of buried land mines in thermal infrared imagery. Results of experimental studies using MWIR (2.2-4.6 $\mu \mathrm{m})$ and LWIR $(8-12 \mu \mathrm{m})$ imaging systems are reported. Thermal clutter due to surface reflected sunlight and skylight are investigated and shown to be the dominant clutter component for both MWIR and LWIR imagery collected during daylight hours. A sensorbased clutter reduction technique, spectral differencing, was considered and found to provide some benefit. The temporal evolution of thermal signatures was investigated. The imagery are found to have near-Gaussian statistics, and therefore the deflection coefficient is a valid measure of detectability. The deflection coefficient for some buried mines was found to improve with time after sunset. In addition, the LWIR band appears to offer some advantages in detection. Clutter mitigation via signal processing is also explored using an "estimator-classifier" technique in which target-related parameters (features) are estimated from the data and detected with a classifier. The theoretical basis of the method is discussed. MWIR and LWIR imagery are used to illustrate both the sensor-based and signalprocessing-based techniques.
\end{abstract}

Keywords: Thermal infrared imagery, clutter, land mines, LWIR, MWIR

\section{INTRODUCTION}

In this work we examine methods for improving detection of buried mines in thermal infrared images. Naturally occurring clutter has long been recognized as a performance limiting factor in IR mine detection. A significant problem in dealing with this clutter is that it derives from several largely independent sources including variations in soil temperature, surface emissivity, and reflected light. These clutter sources are affected by diverse natural phenomena including variations in soil mineral composition and moisture content, inhomogeneous ground cover, the vitality of overlying vegetation, surface topography, and shadowing.

Several techniques for mitigating clutter are considered here. In Section 2 we describe clutter reduction techniques related to sensors and their use. Tests at our facility, reported here, indicate that during daylight hours surfacereflected sunlight and skylight are the dominant clutter sources both at MWIR and LWIR. In Section 2.1 we discuss the nature of reflected-light clutter. Supporting experimental data were acquired on a surrogate mine field constructed at our facility. In Section 2.2 we describe the time evolution of thermal imagery. The behavior of the mine signature and the surrounding soil are compared. In Section 2.3 we describe the concept of spectral differencing, in which surface reflectivity is estimated at one band and used to remove reflected-light clutter at another band. Measurements are presented to illustrate the effectiveness of the concept. Finally, in Section 3 we discuss a signal-processing approach to clutter rejection. The technique presented here is a two-stage process in which a parametric model of the mine signature is fit to the data. The parameters are then used in detection via a classifier trained on measurements. Concluding remarks appear in Section 4.

Send correspondence to B.A.B. (614) 292-0076 (voice), (614) 292-7297 (fax), baertlein.1@osu. edu 


\section{SENSOR-BASED TECHNIQUES}

\subsection{The Role of Reflected Light}

In this section we consider the sources of surface-reflected IR clutter and we present some imagery to illustrate the phenomenon. Some important IR clutter mechanisms are shown in Figure 1. The received radiance $L_{R}\left[\mathrm{~W} \mathrm{~m} \mathrm{~m}^{-2}\right.$ $\mathrm{sr}^{-1}$ ] at an IR sensor can be written as

$$
L_{R}(\lambda, x, y)=\rho(\lambda, x, y) L_{S U N}(\lambda)+\rho(\lambda, x, y) L_{S K Y}(\lambda)+\epsilon(\lambda, x, y) L_{T}(\lambda, x, y),
$$

where $\rho$ is the surface reflectivity, $\epsilon$ is the surface emissivity, $L_{S U N}$ is the radiance due to sunlight and $L_{S K Y}$ is the radiance due to skylight (sunlight scattered by particles and molecules in the earth's atmosphere and thermal radiation from the warm atmosphere). Both $L_{S U N}$ and $L_{S K Y}$ are assumed constant over the surface, i.e., shadowing and surface topography are not considered.

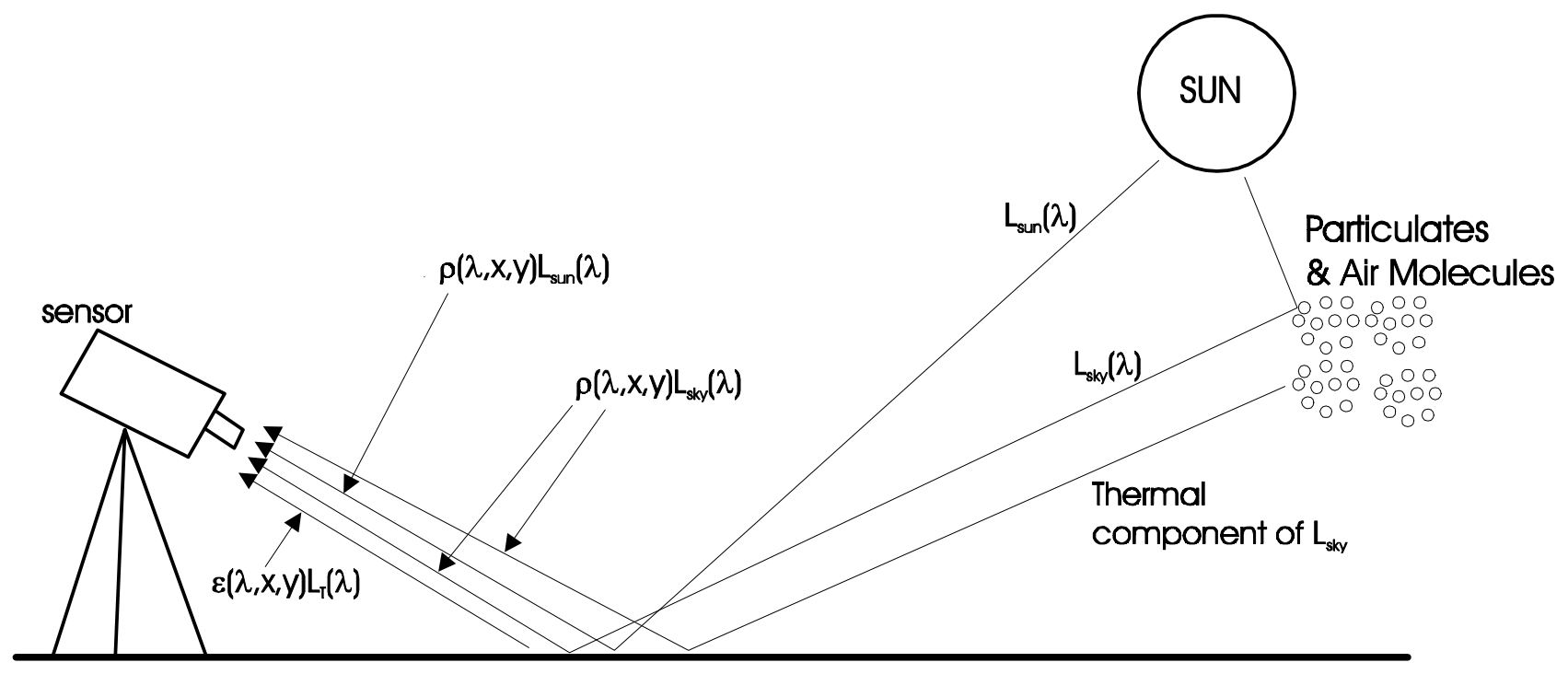

Figure 1. Contributors to a thermal image of the ground include direct sunlight, aerosol-scattered sunlight, thermal emissions from the air reflected by the ground, and thermal emissions from the soil.

For an opaque body that satisfies Kirchhoff's law, we have $\epsilon(\lambda, x, y)=1-\rho(\lambda, x, y)$, and defining

$$
L_{S S}(\lambda)=L_{S U N}(\lambda)+L_{S K Y}(\lambda)
$$

leads to

$$
L_{R}(\lambda, x, y)=[1-\epsilon(\lambda, x, y)] L_{S S}(\lambda)+\epsilon(\lambda, x, y) L_{T}(\lambda, x, y)
$$

Equation (3) illustrates a well known problem in IR detection of buried objects. Even if the incident radiance $L_{S S}$ can be determined by other means, the surface emissivity $\epsilon$ and thermal radiance $L_{T}$ are unknown and vary with surface position. Thus, it is essentially impossible to estimate the desired quantity $L_{T}$ from a single measurement.

A study of the emissivity of natural materials has been presented by Salisbury and D'Aria. ${ }^{1,2}$ The spectral responses of these materials are quite complicated, but in general over the 3-4 $\mu \mathrm{m}$ band rocks and soils exhibit reflectances of $5 \%$ to $30 \%$, while vegetation has reflectances of $2 \%$ to $15 \%$. For the $8-14 \mu \mathrm{m}$ band they found rock reflectances of $1 \%$ to $10 \%$. Vegetation reflectances were found to have approximately the same range.

Experiments were done to study the relative contributions of these illumination components. The experiments were performed at a surrogate minefield emplaced at the ElectroScience Laboratory (ESL) and further described elsewhere. ${ }^{3}$ Some 40 mine-like and clutter-like targets were placed in the native Ohio topsoil and had been in situ for more that 18 months at the time these data were acquired. Surface vegetation on the mine field was completely removed. The test area was not specially conditioned for IR imaging prior to emplacing the targets. As a result, 
Table 1. IR Camera Characteristics.

\begin{tabular}{|c|c|c|}
\hline Parameter & IRRIS 160ST & Agema 1000 \\
\hline \hline Spectral range & MWIR $(2.2-4.6 \mu \mathrm{m})$ & LWIR $(8.0-12.0 \mu \mathrm{m})$ \\
\hline Sensor type & InSb PV array & HgCdTe \\
\hline Array size & $160(\mathrm{~h})$ by $120(\mathrm{v})$ pixels & $590(\mathrm{~h})$ by $401(\mathrm{v})$ pixels \\
\hline FOV & $9.1^{\circ}$ by $6.8^{\circ}$ & NFOV: $5^{\circ}$ by $3^{\circ}$ \\
& & WFOV: $20^{\circ}$ by $13^{\circ}$ \\
\hline IFOV & $1 \mathrm{mrad}$ & NFOV: $.15 \mathrm{mrad}$ \\
& & WFOV: $0.6 \mathrm{mrad}$ \\
\hline Dynamic Range & variable & $100 \mathrm{~K}$ \\
\hline NE $\Delta T$ & $0.025 \mathrm{~K}$ (typ.) $0.040(\mathrm{max})$ & $<0.2 \mathrm{~K}$ \\
\hline Pixel depth & 12 bits & $12 \mathrm{bits}$ \\
\hline
\end{tabular}

there may exist buried objects or voids in this area. The surface of the mine field is planar to within an estimated variation of \pm 2 inches.

As noted above, measurements were performed at different IR spectral bands to better judge the effects of wavelength on clutter. Since the spectrum of sunlight is strongest at visible wavelengths, sensors optimized for long wavelengths will be less affected by direct and reflected sunlight.* A commercial MWIR camera (Cincinnati Electronics IRRIS 160ST) and a LWIR camera (FLIR Sytems Agema 1000) were used in these tests. Sensor characteristics are shown in Table 1. The MWIR sensor offers a InSb array detector with low internally generated sensor noise, expressed as the noise equivalent temperature difference $(\mathrm{NE} \Delta \mathrm{T})$. This metric determines the weakest detectable signal. The LWIR camera was borrowed from the US ARMY Night Vision Laboratory for the duration of this test. This sensor is a second-generation scanning array. The spectral range of its HgCdTe detector is 8-12 $\mu \mathrm{m}$ and it produces 12 bit data with a dynamic range of $100 \mathrm{~K}$. It also has the ability to switch between two fields of view.

We first illustrate the relative contributions of direct sunlight and skylight through shadowing for the MWIR sensor. We compared images of regions illuminated by direct sunlight and shadowed (i.e., without direct illumination). Figure 2 shows data collected at 11:55 on 25 July 1998. Figures 2(a) and (b) illustrate the shadowed and unshadowed IR images of the field, while Figure 2(c) shows the positions of fiducial markers, mines, and a flush-buried concrete piling. The mines in this scene are seven-inch plastic disks buried one inch in the soil. We find that it is nearly impossible to distinguish the fiducial markers and mines due to heavy clutter in the unshadowed image. There is less clutter in the shadowed image and the fiducial markers can be easily seen, but the mine signatures are not apparent. On the basis of these data we conclude that direct illumination of the scene by sunlight is the dominant MWIR clutter source, and skylight (both aerosol-scattered sunlight and thermal emission by the air) is also a significant clutter source.

The most dramatic illustration of the importance of surface-reflected light on MWIR imagery occurs when the sensor is operated at night. Figure 3 shows the results of an experiment performed on 9 June 1998 in which data were collected after sunset. Figure 3(a) shows the fiducial marker geometry, the mine positions and the concrete pilings. Figures 3(b)-(f) are the IR imagery taken at 19:10, 20:25, 21:40, 23:10 and 00:40, respectively. In these figures the fiducial marker positions are obvious, and one can also identify the mine signatures. It is evident that MWIR imagery is best captured at night to avoid surface-reflected light. Operating at night does not remove all reflected clutter, since the night sky continues to produce thermal radiation in the infrared region, which is reflected from the ground.

The role of surface-reflected light in LWIR sensors is explored in Figure 4. Figure 4(a) illustrates the region of interest including the mine positions, fiducial markers and the concrete pilings. Figure 4(b) shows the data collected on 13 October 1998 at 14:30. The direct sunlight contribution is present in this experiment. Although the fiducial markers can be distinguished, it is not possible to identify the mine positions due to heavy clutter. In Figure 4(c) data collected on 13 October 1998 at 18:00 are presented. This image is captured just after the whole mine-field is completely shadowed by an adjacent building. The image is less cluttered and it is now possible to identify the mine positions. In Figure 4(d) we present an image captured on 12 October 1998 after sunset at 21:00. In this figure the

\footnotetext{
* Using a $6000 \mathrm{~K}$ blackbody radiator as a model for solar radiation, we find that the solar radiance at $3 \mu \mathrm{m}$ will be roughly two orders of magnitude stronger than at $10 \mu \mathrm{m}$. In addition, the reflectivity of soil is roughly an order of magnitude larger at $3 \mu \mathrm{m}$ than at $10 \mu \mathrm{m} .^{2}$
} 
mines can be clearly identified. These results show that surface-reflected light is also a major source of clutter in LWIR imagery. Figure 5 shows a sequence of images taken after sunset.

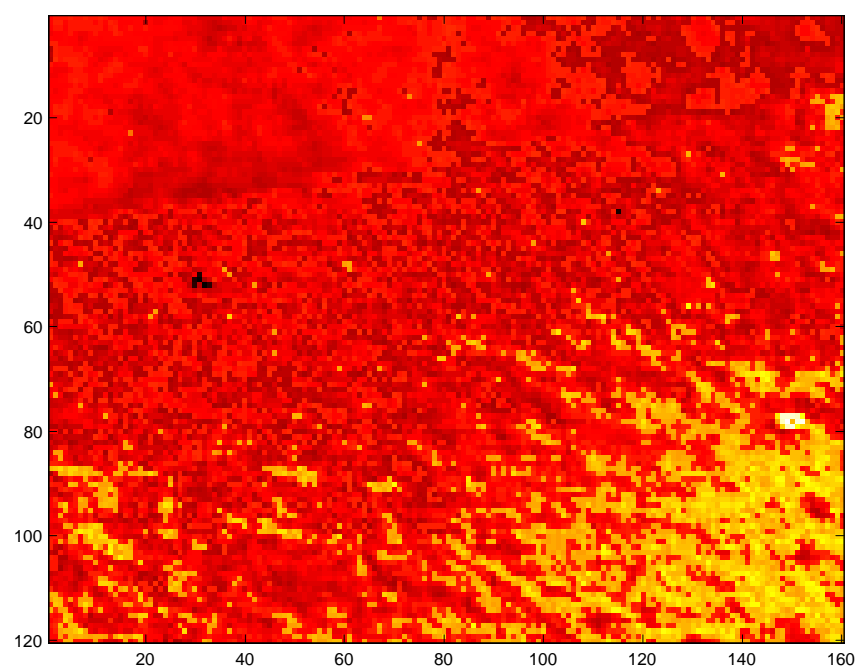

(a)

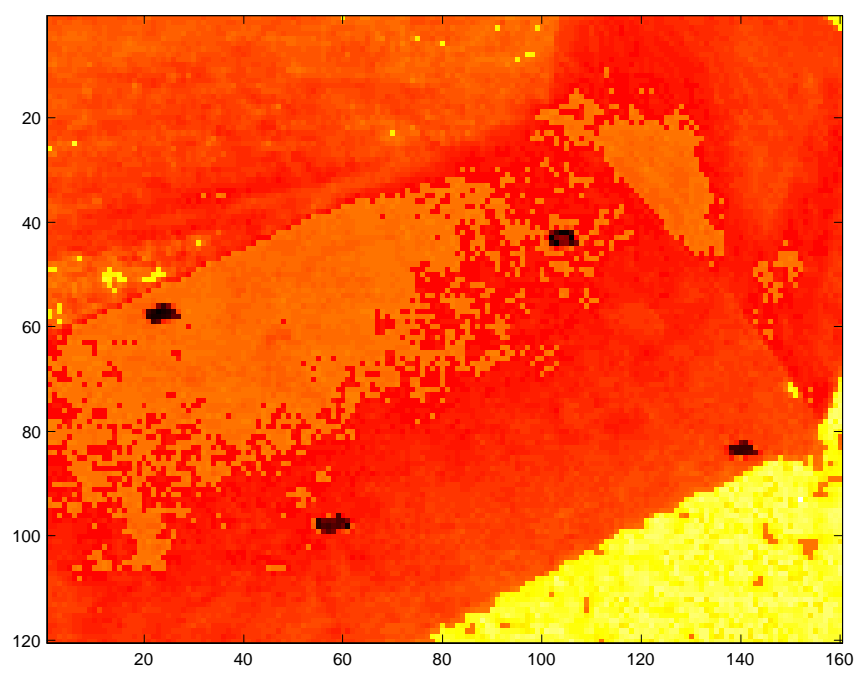

(b)

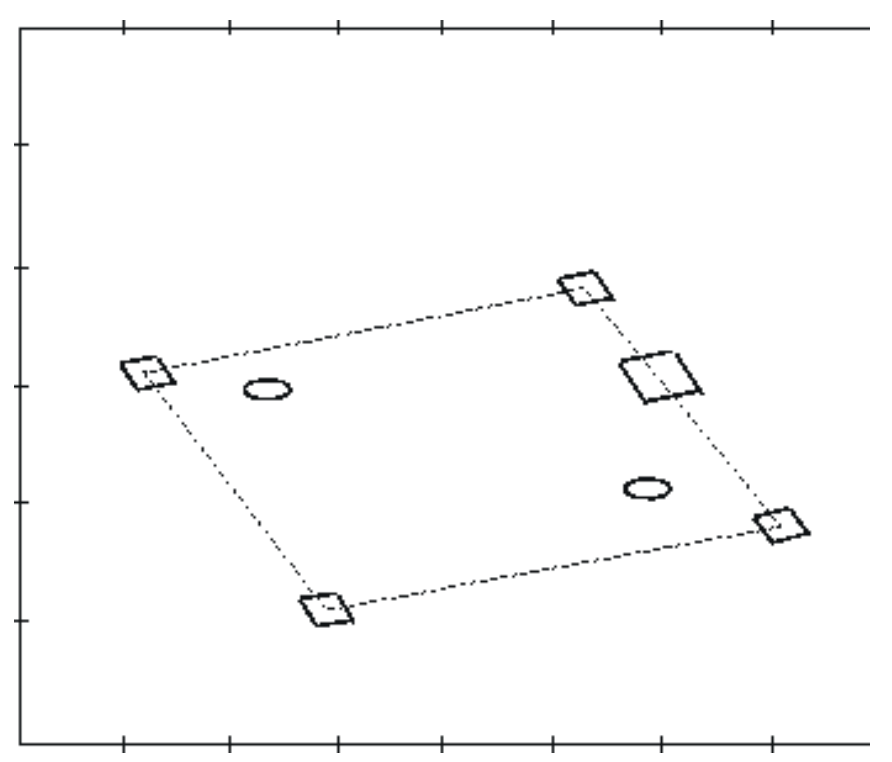

(c)

Figure 2. MWIR images collected on 25 July 1998 at 11:55 showing (a) unshadowed IR imagery, (b) shadowed IR imagery, and (c) a sketch of the region of interest including the mine positions (shaded circles), fiducial markers (small squares) and a flush-buried concrete piling (large square). 


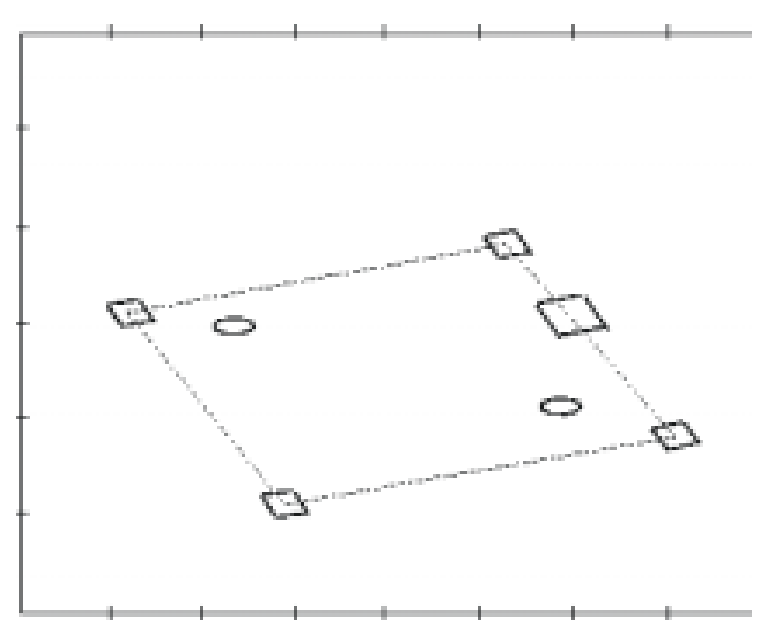

(a)

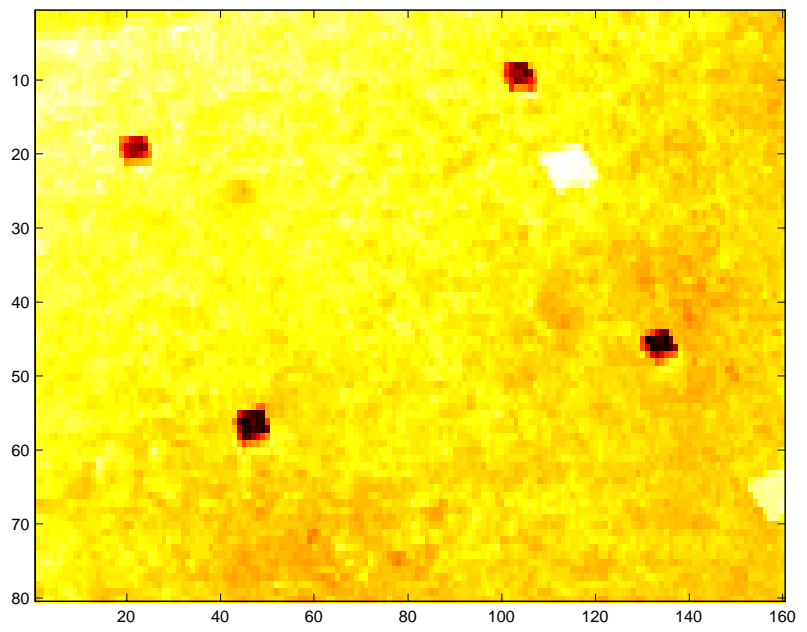

(c)

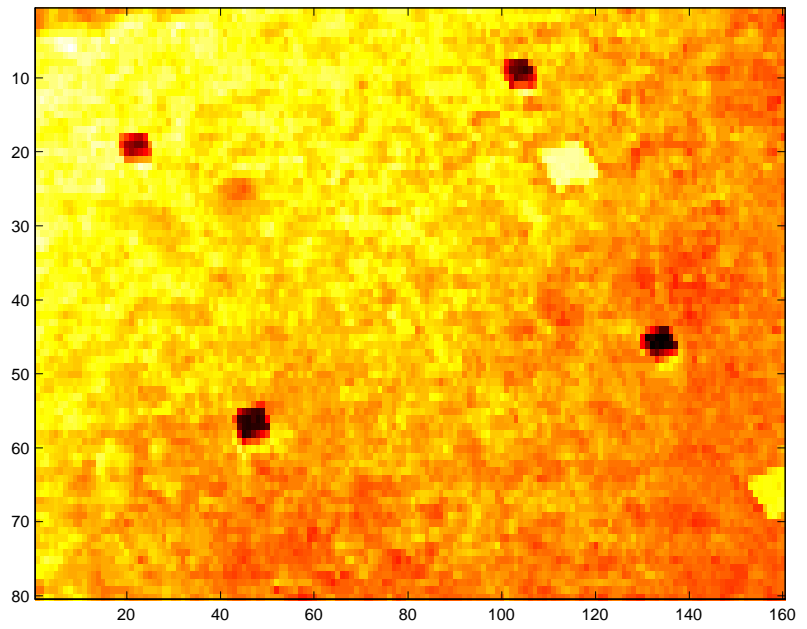

(e)

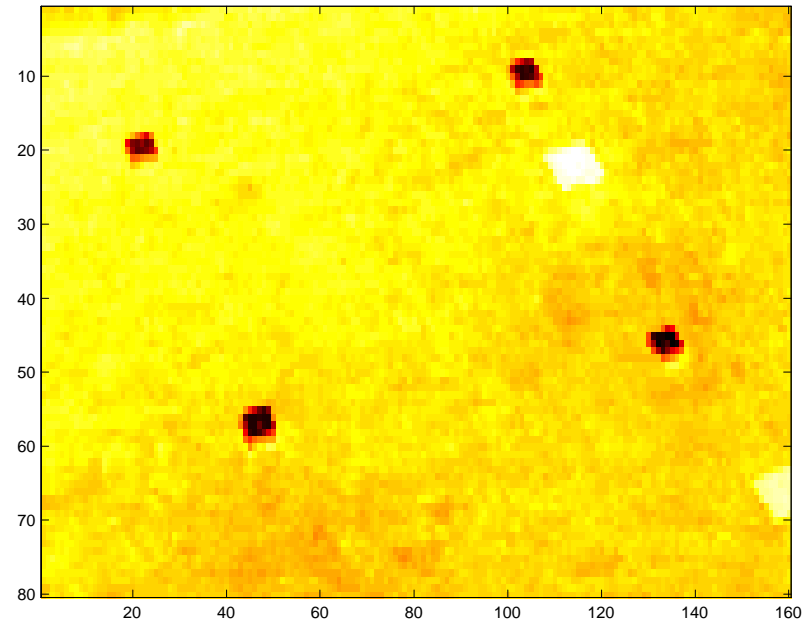

(b)

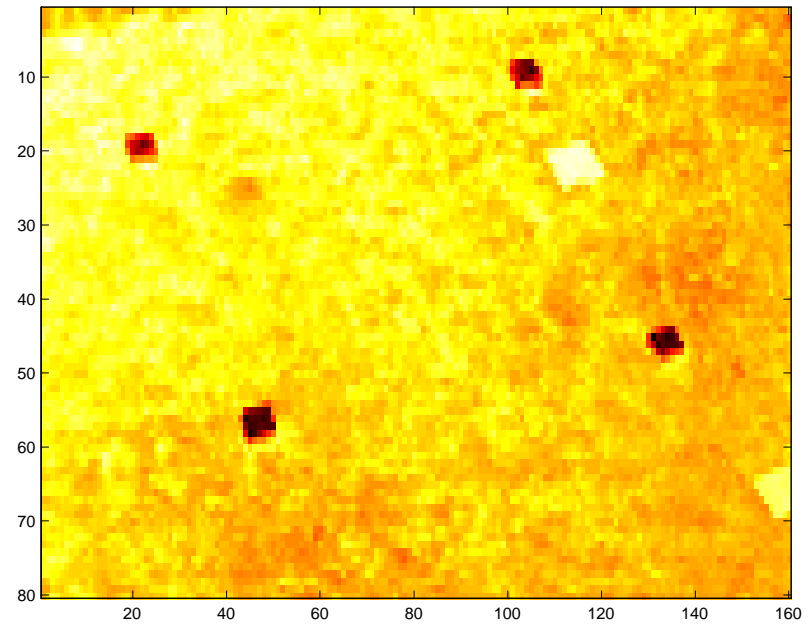

(d)

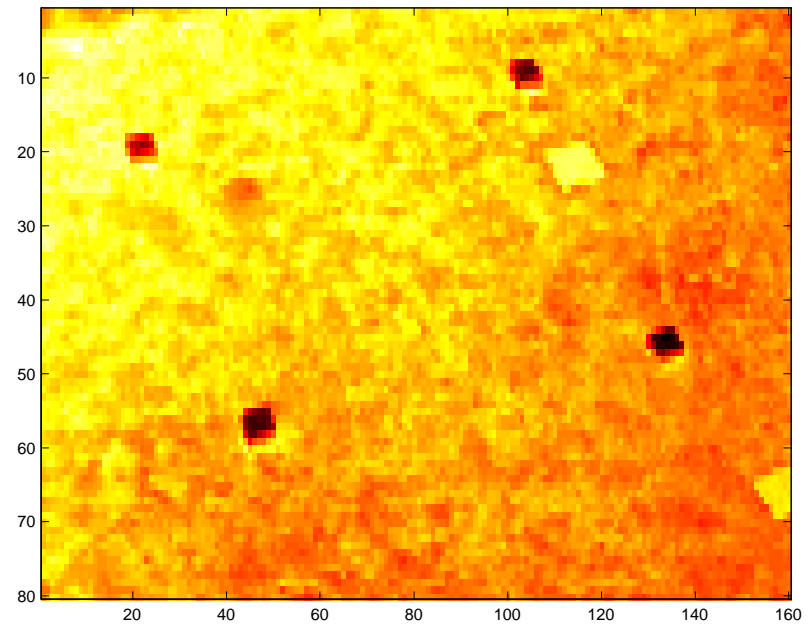

(f)

Figure 3. MWIR images collected on 9 June 1998. (a) Sketch of the region of interest including the mine positions, fiducial markers and the concrete pilings. Images were captured at (b) 19:10, (c) 19:25, (d) 20:10. (e) 20:40, and (f) 21:40. 


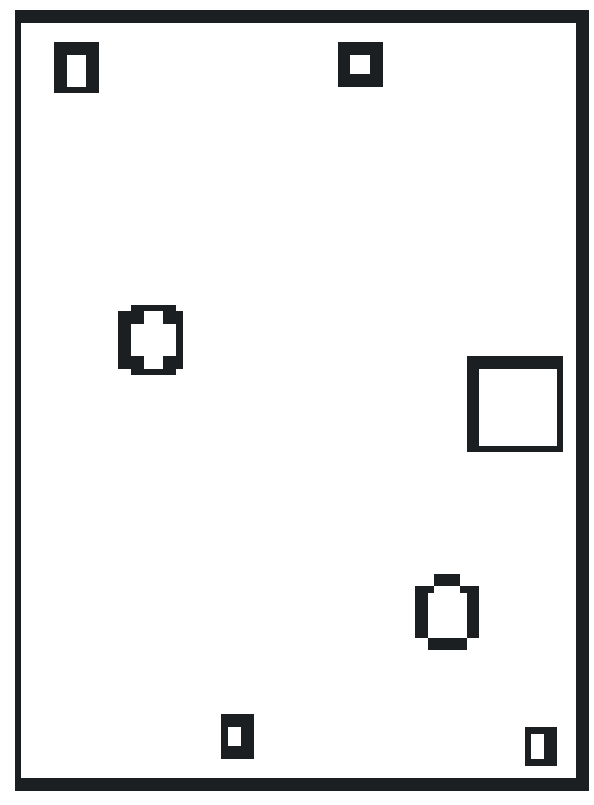

(a)

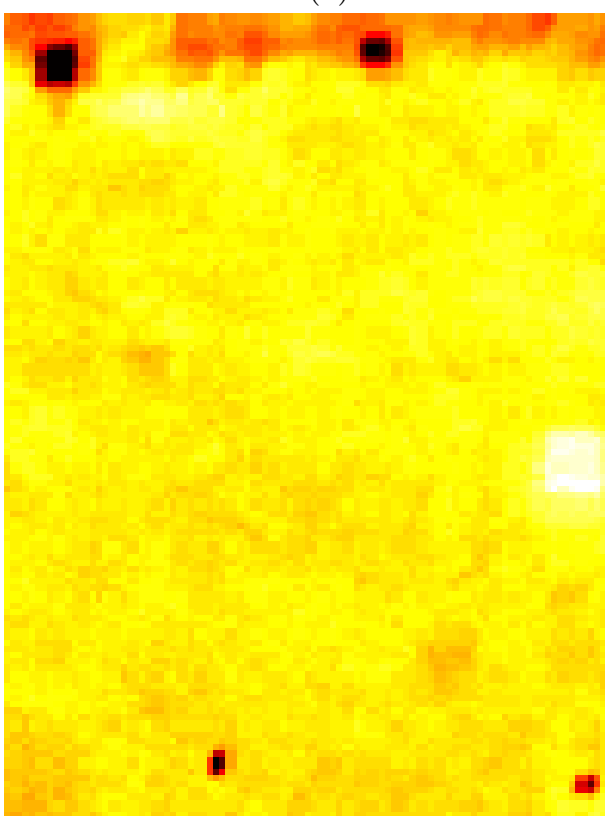

(c)

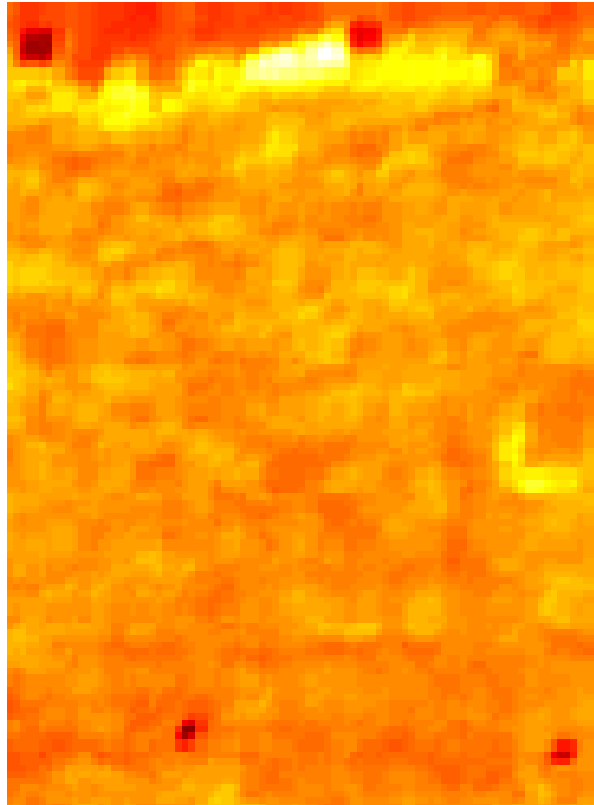

(b)

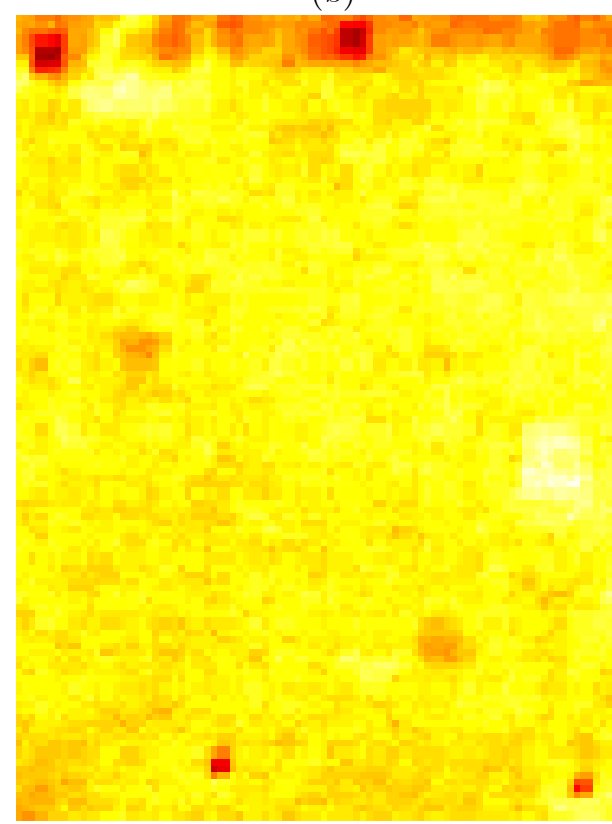

(d)

Figure 4. LWIR images captured 13 October 1998. (a) Sketch of the region of interest including the mine positions, fiducial markers and the concrete pilings. Images were captured at (b) 14:30, (c) 18:00, and (d) 21:00. 


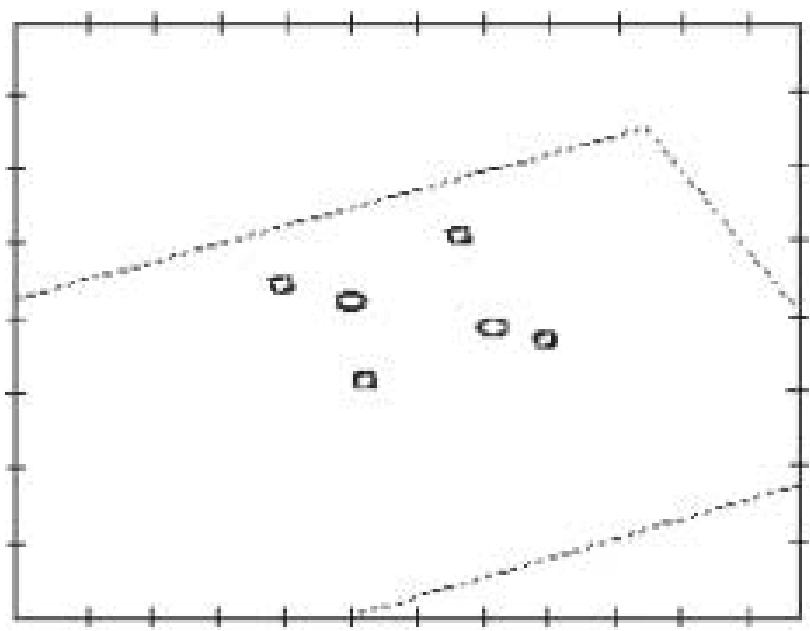

(a)

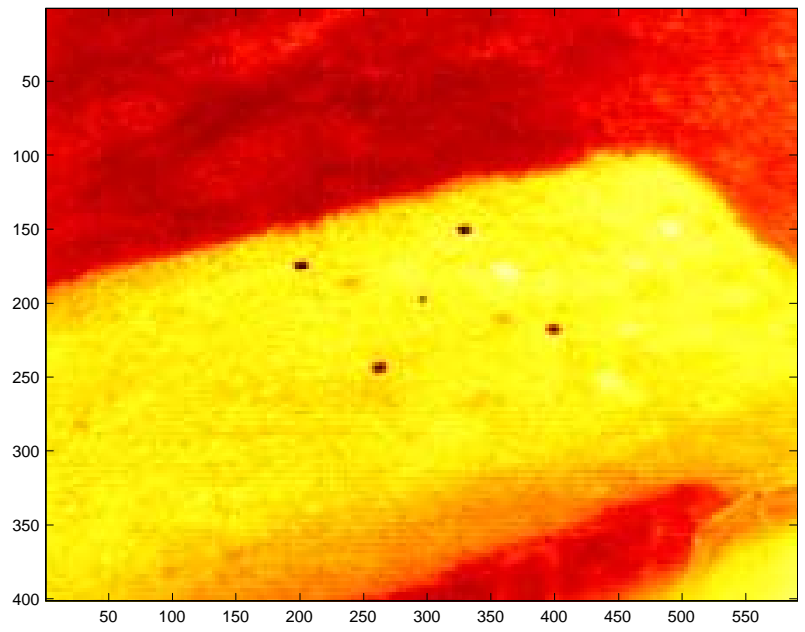

(c)

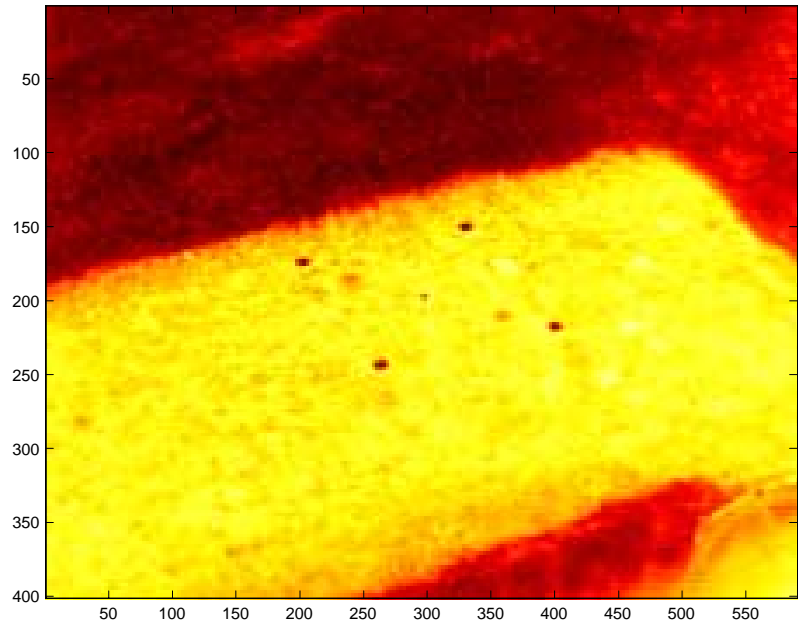

(e)

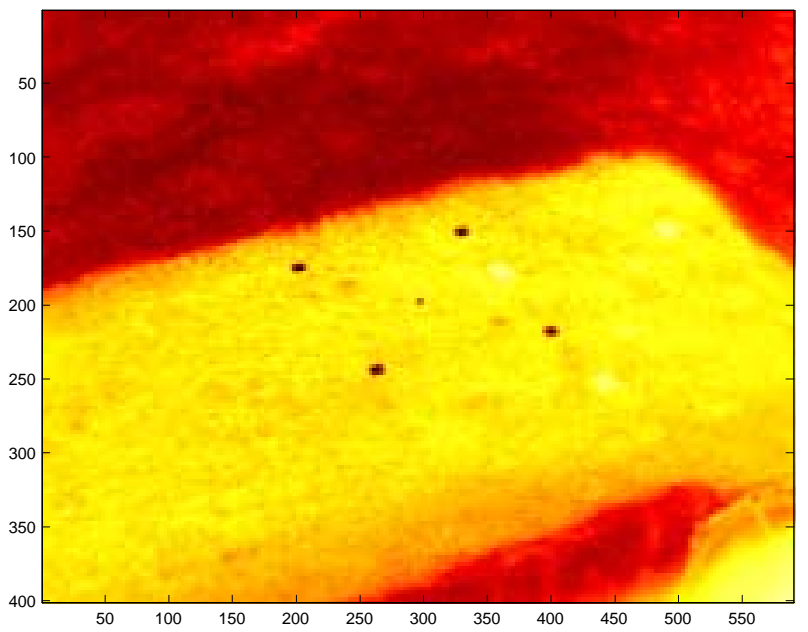

(b)

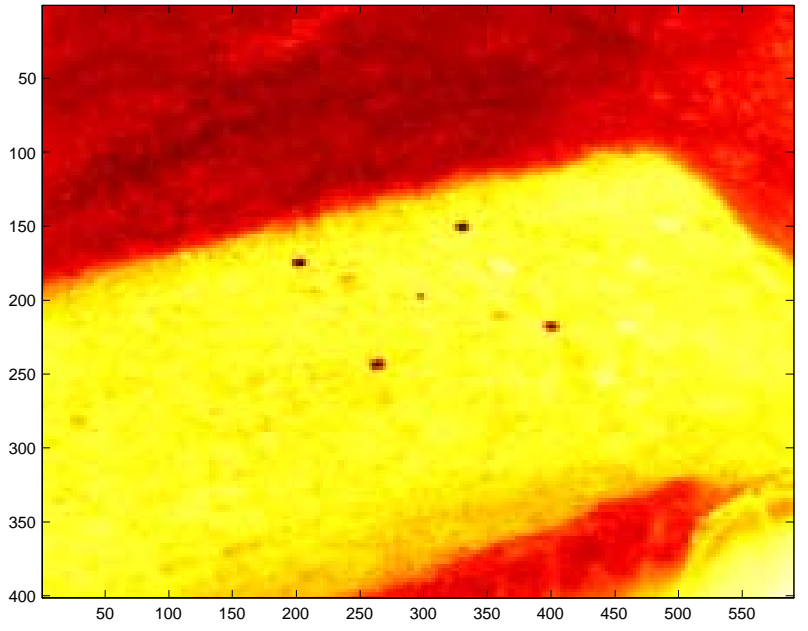

(d)

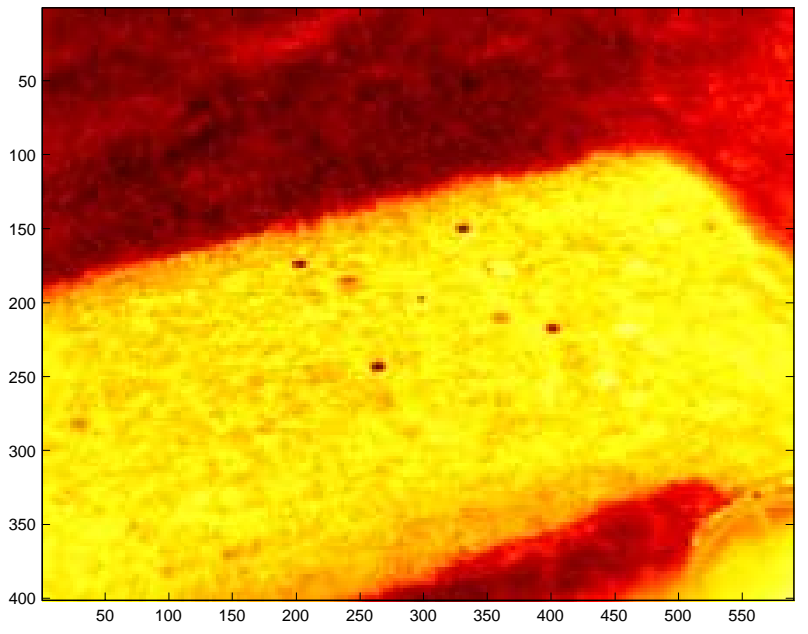

(f)

Figure 5. LWIR images captured 30 October 1998. (a) A sketch of the mine area showing the positions of mine surrogates and fiducial markers. Images were captured at (b) 18:10, (c) 19:10, (d) 20:10, (e) 21:40, and (f) 22:40. 


\subsection{Temporal Evolution Studies}

The data displayed in Figures 3 and 5 were part of a study undertaken to identify time-dependent differences in the thermal behavior of soil over a mine with respect to the surrounding soil. Those images indicate that thermal mine signatures are dynamic phenomena and, in principle, their evolution is described by thermal models. In humanitarian demining we have the luxury of being able to acquire the time history of the minefield thermal imagery, which can provide additional information leading to improved detection.

The temperature of soil is constantly changing throughout the scene, and any mine-related temperature differences must be detected in the presence of this background change. To minimize the number of clutter phenomena present, the images are acquired after sunset. The timing of the measurements with respect to sunset is, however, an important factor as shown in the preceeding section. We collected image sequences at regularly spaced time intervals using both the MWIR and LWIR cameras. Software was developed to control the cameras and to automatically acquire imagery every 15 minute for several hours.

MWIR images were collected on 9 July 1998 starting at 19:10 (near sunset) and continuing for more than four hours. Representative samples of the data were shown in Figure 3. It can be seen that the clutter in the scene increases as time increases. The reason for this change has not been conclusively determined, but it is possible that small protruding surface features cool more rapidly than larger flat ground regions, because of the greater exposed surface area of the former.

Histograms of these images (not shown here) appear Gaussian. A test of this condition is possible by using the Kolmogorov-Smirnov (KS) statistic, ${ }^{7}$ which permits us to determine the probability that data is drawn from a specified distribution. For these images typical levels of significance for the test are near unity, indicating a near-Gaussian distribution for the image pixels.

LWIR imagery was acquired 30 October 1998 starting at 19:10. Representative results, acquired between 18:10 and 22:40, were shown in Figure 5. The histograms of the LWIR imagery are strongly bimodal as a result of imaging both bare soil (over the mine field) and surrounding vegetation, but imagery with a single type of ground cover appears to have near-Gaussian statistics also.

It is well known that the detection and false alarm rates of targets in Gaussian noise are directly related to the "deflection coefficient" $d^{2}=\left(\mu_{1}-\mu_{0}\right)^{2} / \sigma^{2}$, which is analogous to the signal-to-noise ratio. In this expression $\mu_{1}$ is the mean of the signal with the target present, $\mu_{0}$ is the mean of the signal with the target absent, and $\sigma_{0}^{2}$ is the variance of the signal with the target absent. The deflection coefficient was computed for the two mines present in each of the MWIR and LWIR data shown in Figures 3 and 5, and the result is shown in Figure 6. We find that the deflection coefficient is generally increasing with time in both bands, which suggests that detection will be improved later in the evening. The MWIR data show inconsistent trends, and the LWIR data have markedly higher values. These limited data suggest that the LWIR band may offer some advantages for thermal mine detection.

\subsection{Spectral Differencing}

In deriving equation (3) we observed that reflected light is a significant source of clutter. Furthermore, even if $L_{S S}$ could be determined, this equation cannot be used to estimate $L_{T}$, since the surface emissivity $\epsilon$ is unkown. In principle, two measurements can be used to eliminate the $L_{S S}$ contribution in a straightforward manner. Another approach to dual-band operation was explored previously in a series of papers by Del Grande et al. ${ }^{4,5,5}$ In that work it was shown that a ratio of measurements at two bands was directly related to soil temperature, although the effect of surface-reflected light was apparently ignored. We make the key assumption that although the emissivity may vary strongly with position, its wavelength variation is essentially independent of position. ${ }^{4}$ Thus, the spatial and spectral variation of the emissivity can be factored as follows:

$$
\rho(\lambda, x, y) \approx \rho(\lambda) A(x, y)=[1-\epsilon(\lambda)] A(x, y)
$$

where $A(x, y)$ is an unknown function that is independent of $\lambda$. Given measurements of a region at two wavelengths,

$$
L_{R}\left(\lambda_{j}, x, y\right)=\rho\left(\lambda_{j}\right) A(x, y) L_{S S}\left(\lambda_{j}\right)+\epsilon\left(\lambda_{j}\right) A(x, y) L_{T}\left(\lambda_{j}, x, y\right) \quad j=1,2
$$

we form the weighted difference image

$$
\Delta L_{R}(x, y) \equiv L_{R}\left(\lambda_{1}, x, y\right)-C\left(\lambda_{1}, \lambda_{2}\right) L_{R}\left(\lambda_{2}, x, y\right)
$$




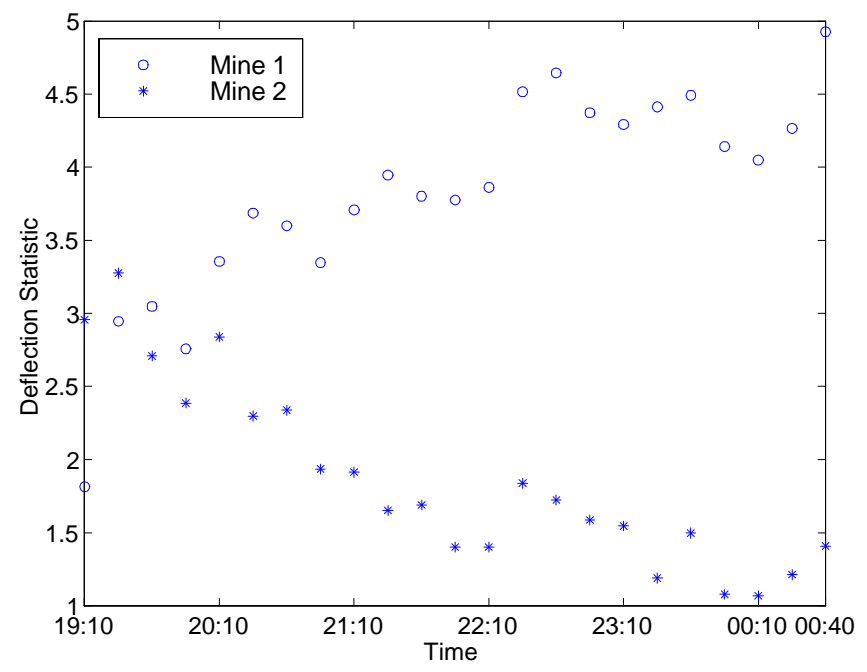

(a)

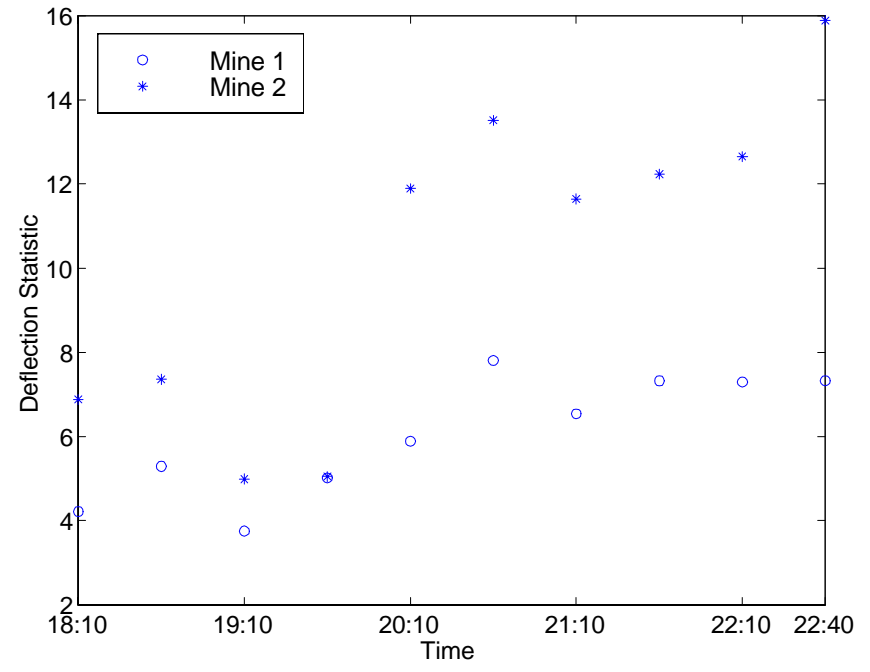

(b)

Figure 6. The deflection coefficient measured by (a) MWIR and (b) LWIR cameras as a function of time.

where $C$ is a constant. If we choose

$$
C\left(\lambda_{1}, \lambda_{2}\right)=\frac{\rho\left(\lambda_{1}\right) L_{S S}\left(\lambda_{1}\right)}{\rho\left(\lambda_{2}\right) L_{S S}\left(\lambda_{2}\right)}
$$

then we have

$$
\Delta L_{R}(x, y)=A(x, y)\left[\epsilon\left(\lambda_{1}\right) L_{T}\left(\lambda_{1}, x, y\right)-C\left(\lambda_{1}, \lambda_{2}\right) \epsilon\left(\lambda_{2}\right) L_{T}\left(\lambda_{2}, x, y\right)\right]
$$

in which there is no contribution from the direct radiation. If one wavelength, say $\lambda_{2}$, is in the visible range, then this formulation simplifies slightly. We have $L_{T}\left(\lambda_{2}, x, y\right) \approx 0$ for $T \approx 300 K$, and

$$
\Delta L_{R}(x, y)=A(x, y) \epsilon\left(\lambda_{1}\right) L_{T}\left(\lambda_{1}, x, y\right)
$$

Thus, we find that a weighted form of the thermal radiation $\epsilon L_{T}$ can be determined by an appropriately weighted difference of visible and IR imagery. The appropriate value of $C$ is seen to depend on environmental factors which are not easily measured. In this work we vary $C$ to achieve a value that is most effective in suppressing clutter.

To explore the spectral differencing concept MWIR and LWIR images were combined. The imagery was acquired on 5 November 1998 after sunset to avoid surface-reflected clutter. The objective of differencing under these conditions is to remove the reflected radiance from the warm atmosphere. Figures 7 (a) and (b) show the original MWIR and LWIR imagery, respectively. Figures 7 (c)-(e) illustrate the compensated IR images for different $C$ values. Increasing the value of the $C$ increase the influence of the LWIR image. The results show that it is possible to reject a modest amount of clutter by choosing $C$ apppropriately. Figures 7 (c)-(e) illustrate the trade-off between the maximum contrast and the minimum clutter. If we increase $C$ further, we continue to decrease the clutter, but we also decrease the mine signature. Figure 7 (f) shows the ratio of these bands as suggested by LeSchack and Del Grande. ${ }^{4}$ We find the performance of these methods to be almost indistinguishable.

\section{ESTIMATOR-CLASSIFIER DETECTION}

We also explored clutter reduction through image processing. Efforts described here show that image processing can provide an additional detection improvement beyond that achievable by sensor-based techniques.

IR signatures depend on a variety of unknown factors including target depth, target size, soil conditions, and thermal history. Thus, detection algorithms which require a known signature (e.g., matched filtering) are inappropriate. We will consider the more general case in which the target can be modeled by a known function $\mathbf{G}(\mathbf{R} ; \boldsymbol{\Theta})$ with unknown parameters $\boldsymbol{\Theta}$. In this case we express the measured data $\mathbf{r}$ as

$$
\begin{aligned}
& \mathcal{H}_{0}: \mathbf{r}(\mathbf{R})=\mathbf{G}(\mathbf{R} ; \boldsymbol{\Theta})+\mathbf{n}(\mathbf{R}) \\
& \mathcal{H}_{1}: \mathbf{r}(\mathbf{R})=\mathbf{n}(\mathbf{R})
\end{aligned}
$$




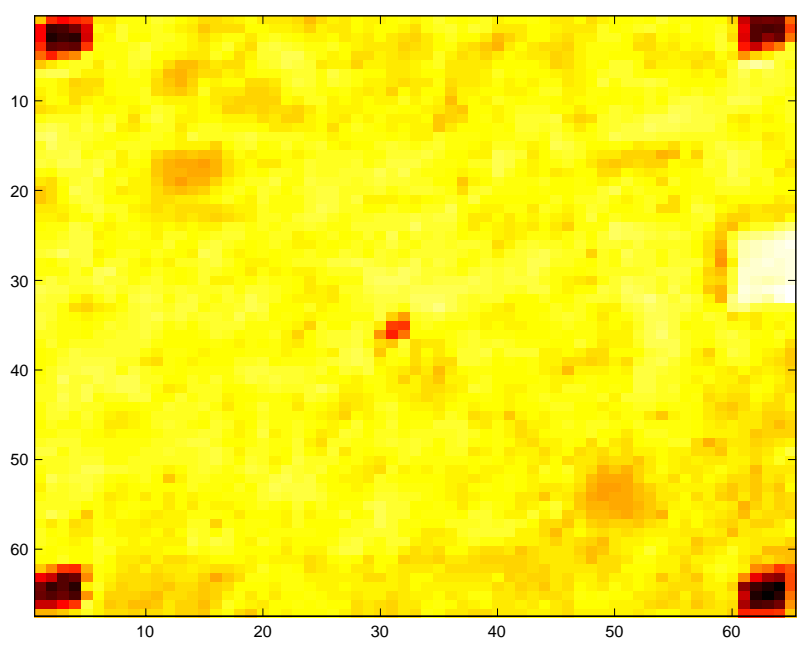

(a)

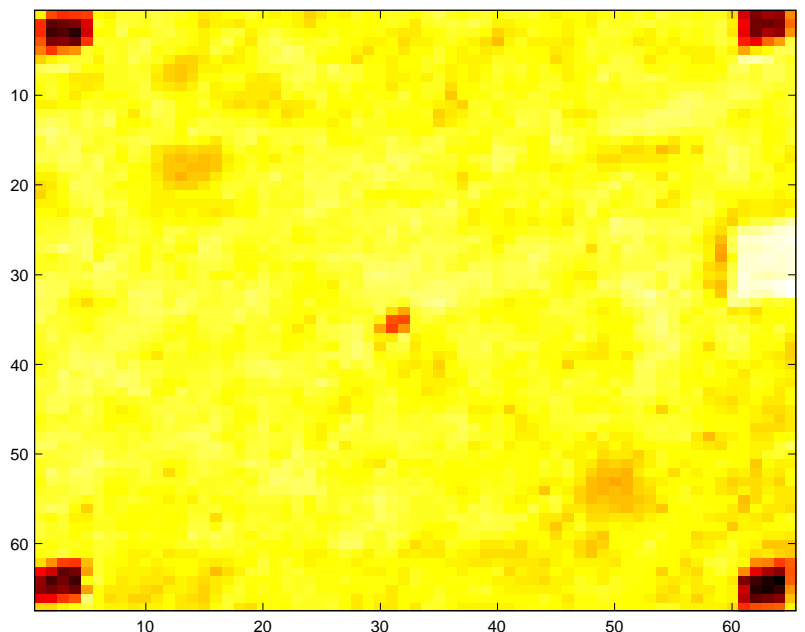

(c)

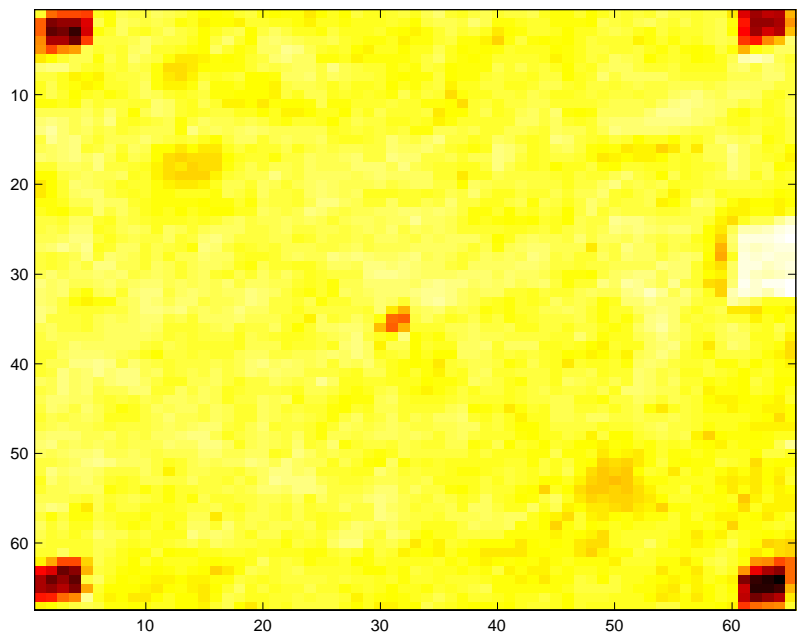

(e)

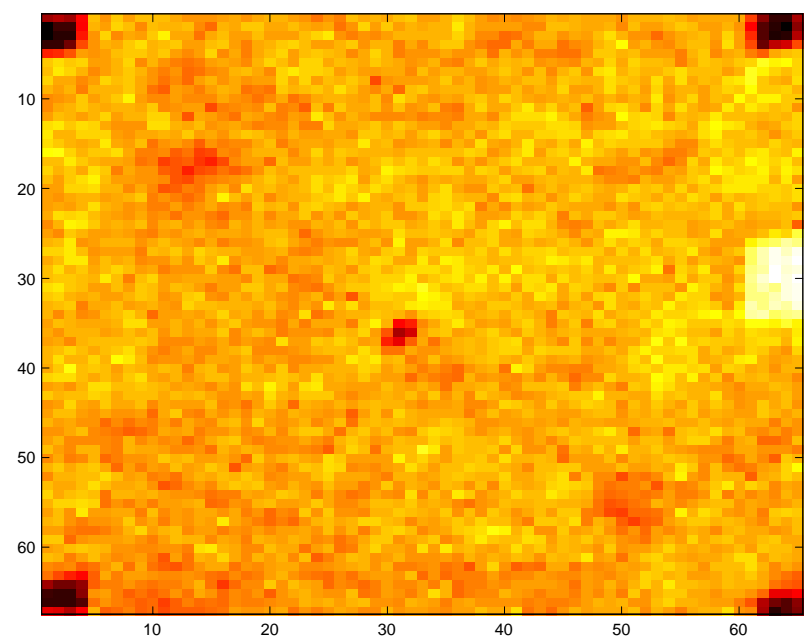

(b)

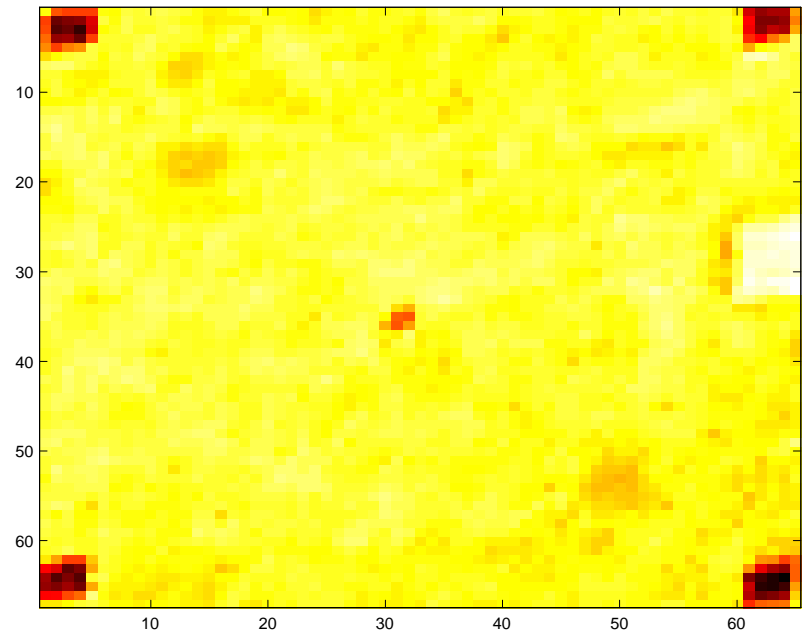

(d)

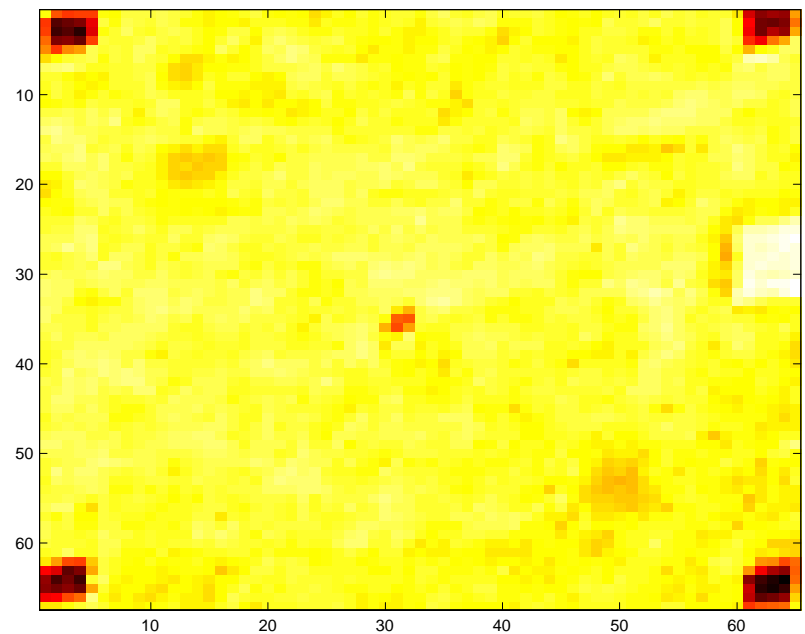

(f)

Figure 7. (a) The original MWIR image. (b) The original LWIR image. Compensated images for (c) $C=0.03$, (d) $C=0.05$, and (e) $C=0.07$. Figure (f) shows the ratio of the MWIR and LWIR bands as suggested by LeSchack and Del Grande. 

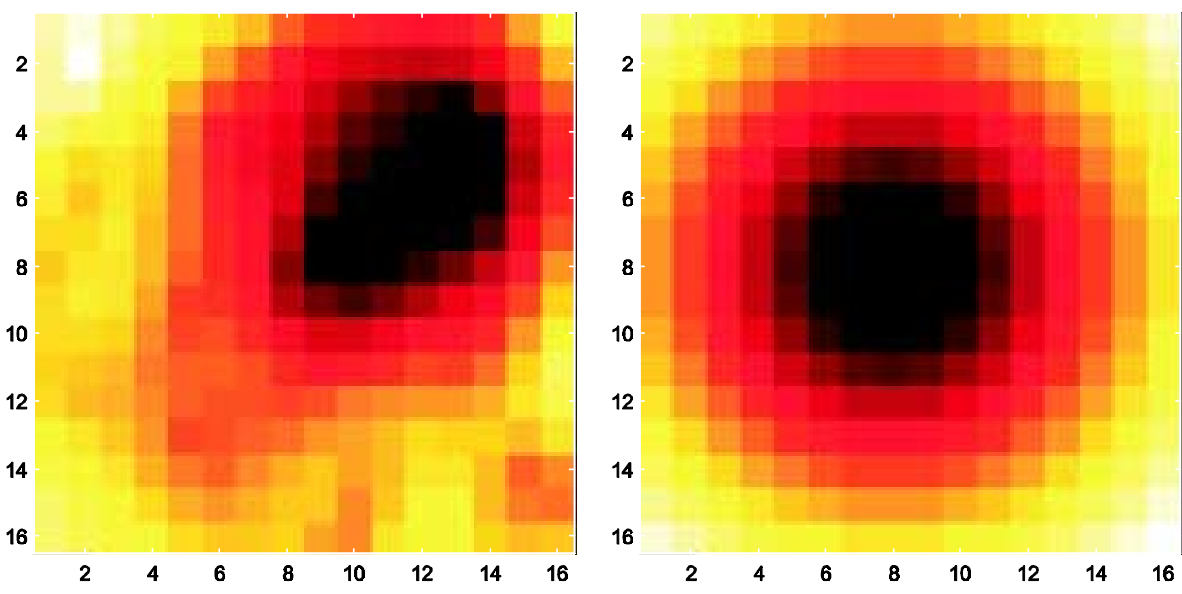

Figure 8. An example mine signature acquired with the MWIR camera (left) and the simulation G (right).

We assume that the components of $\mathbf{n}$ are jointly normal random variables with zero mean and covariance matrix $\mathbf{C}_{n}$.

For this composite hypothesis, we employ a generalized likelihood ratio test which involves $\hat{\boldsymbol{\Theta}}$, the maximum likelihood estimate of the unknown parameter $\Theta$ given by $\hat{\Theta}=\arg \min _{\Theta} \mathcal{L}$. In this result the $\log$-likelihood $\mathcal{L}$ is given by

$$
\mathcal{E}=[\mathbf{r}(\mathbf{R})-\mathbf{G}(\mathbf{R} ; \boldsymbol{\Theta})]^{T} \mathbf{C}_{n}^{-1}[\mathbf{r}(\mathbf{R})-\mathbf{G}(\mathbf{R} ; \boldsymbol{\Theta})]
$$

Selection of the model $\mathbf{G}$ is difficult in this case, since little is known about thermal IR mine signatures. Based on empirical data we propose the following function:

$$
\mathbf{G}(\mathbf{R} ; \boldsymbol{\Theta})=I_{\text {back }}+\frac{I_{\text {mine }}}{\left(1+\left(\frac{\mathbf{R}-\mathbf{R}_{0} \mid}{\alpha}\right)^{\beta}\right)}
$$

where $\mathbf{R}$ is the position of the image pixel, $\mathbf{R}_{0}$ is the position of the mine, and the parameter vector $\boldsymbol{\Theta}$ is given by

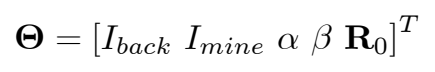

where $I_{\text {back }}$ represents the background amplitude, $I_{\text {mine }}$ represents the amplitude of the mine signal, $\alpha$ represents the size of the mine, and $\beta$ represents the exponent of a power-law decay rate for the signature. With four parameters there is sufficient generality in this model to allow it to fit most targets. Not all of the parameters in $\Theta$ are relevant to detection of the mine. We find that the parameters of greatest interest are $\left(I_{\text {mine }}-I_{\text {back }}\right), \alpha, \beta, \operatorname{and} \log (\mathcal{L})$. An example IR mine signature and the simulated signature $\mathbf{G}$ are shown in Figure 8.

We evaluated the performance of this algorithm on the surrogate mine field noted previously. ${ }^{3}$ In Figures 9 the ROC curves obtained using the estimator-classifier approach are presented. In the left figure only the IR detectable targets (plastic mine surrogates and voids with diameter greater than a few inches) are declared to be valid detections. The classifier readily learns this class of target as shown by the excellent performance. Detection of all mine surrogates is a more challenging task as shown in the right figure. Many mine surrogates, because of their size or thermal properties, offer weak thermal signatures. This fact has motivated the use of sensor fusion, as we describe elsewhere. ${ }^{3}$

\section{CONCLUSION}

In this paper we have documented several investigations directed toward improving detection of buried mines in thermal IR imagery. We found that for both MWIR and LWIR sensors, surface-reflected radiation comprises the dominant source of daytime image clutter. Shadowing of the scene produced some benefit at MWIR and LWIR, but significant reflections are still seen in MWIR from aerosol-scattered sunlight and thermal radiation from the warm atmosphere. MWIR imagery acquired at night shows dramatically reduced clutter. For LWIR the benefits of night versus shadowed imagery are not as dramatic, but they are significant nonetheless. We showed that clutter can also 

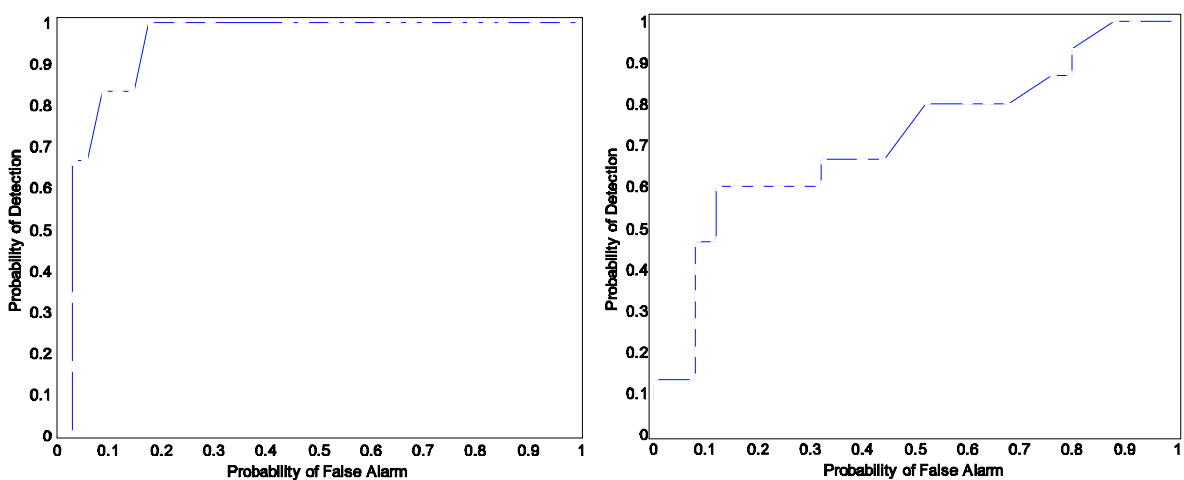

Figure 9. ROC curves obtained using the estimate-classify approach. For the curve on the left, only IR detectable objects (mine surrogates and voids larger than a few inches) were declared to be valide detections. For the curve on the right, only mine surrogates are declared to be valid detections.

be suppressed by using multi-spectral data. By forming a weighted difference of two images of the same scene it is possible to increase enhance the mine signature somewhat. Studies of the time evolution of thermal imagery suggest that the ability to detect buried mines in thermal imagery increases with time after sunset. The LWIR band appears to offer some advantages in detection.

Signal processing approaches to the mine detection problem were also considered. We developed a detection algorithm based on pattern recognition principles. In this approach signature parameters are computed and used in a classifier. The ROC curve for this approach was computed and found to be good for certain classes of mines, however, other mine types (i.e., those smaller than about three inches and those with significant metal content) were poorly detected in our tests.

\section{ACKNOWLEDGMENTS}

The authors would like to thank the US Army Night Vision Laboratory and John Moulton of E-OIR for the loan of their Agema camera. This project was supported by funds from Duke University under an award from the ARO (the OSD MURI program). The findings, opinions and recommendations expressed therein are those of the authors and are not necessarily those of Duke University or the ARO.

\section{REFERENCES}

1. Salisbury, J.W. and D. M. D'Aria, "Emissivity of terrestrial materials in the 8-14 $\mu$ m atmospheric window," Remote Sensing Environment, Vol. 42, pp. 83-106, 1992.

2. Salisbury, J.W. and D. M. D'Aria, "Emissivity of terrestrial materials in the $3-5 \mu \mathrm{m}$ atmospheric window," Remote Sensing Environment, Vol. 47, pp. 345-361, 1994.

3. Gunatilaka, A. and B. A. Baertlein, "Comparison of pre-detection and post-detection fusion for mine detection," in Detection and Remediation Technologies for Mines and Minelike Targets IV, J. T. B. A. C. Dubey, J. F. Harvey and R. E. Dugan, eds., SPIE 3710-118, 1999.

4. LeSchack, L. A., and N. K. Del Grande, "A dual-wavelength thermal infrared scanner as a potential airborne geophysical exploration tool," Geophys., 41(6), p. 1318-1336, 1976.

5. Del Grande, N. K., G. A. Clark, P. F. Durbin, D. J. Fields, J. E. Hernandez, and R. J. Sherwood, "Buried object remote detection technology for law enforcement," Surveillance Technol., Proc. SPIE 1479, pp. 335-351, 1991.

6. Del Grande, N. K., P. F. Durbian, M. R. Gorvad, D. E. Perkins, G. A. Clark, J. E. Hernandez, and R. J. Sherwood, "Dual-band infrared capabilities for imaging buried object sites," in Underground and Obscured Object Imaging and Detection, N. K. Del Grande et al. (eds.), Proc. SPIE 1942, pp. 166-177, 1993.

7. Kendall, M. G., Kendall's Advanced Theory of Statistics: Volume 2, Classical Inference and Relationship, 5th ed., Oxford University Press, New York, NY, 1991. 